Check for updates

Cite this: RSC Adv., 2018, 8, 24458

Received 23rd May 2018

DOI: $10.1039 / c 8 r a 04401 e$

rsc.li/rsc-advances
Accepted 28th June 2018

\section{Excellent binding effect of L-methionine for immobilizing silver nanoparticles onto cotton fabrics to improve the antibacterial durability against washing}

\author{
Jing Zhou, ${ }^{a}$ Dongrong Cai, ${ }^{a}$ Qingbo Xu, ${ }^{a}$ Yanyan Zhang, ${ }^{a}$ Feiya Fu, ${ }^{a}$ Hongyan Diao ${ }^{\text {bb }}$ \\ and Xiangdong Liu (D) *a
}

Silver nanoparticles (Ag NPs) have outstanding antimicrobial effects, but their weak adhesive force onto cotton fiber surfaces often causes undesired silver loss from antibacterial fabrics, diminishing antibacterial durability, and even leading to environmental and health risks. To improve adhesion of the Ag NPs, various strategies have been tried, but achieving long-term antibacterial effectiveness still remains challenging. Here, L-methionine is proposed as a binder reagent because it has low toxicity towards mammalian cells and has a methyl group to enhance its coordination ability. The antibacterial cotton fabric was fabricated via a very simple pad-dry-cure process: after dipping a cotton fabric in an Lmethionine solution followed with heating for esterification, Ag NPs are formed via the reaction of silver nitrate with sodium borohydride. The resulting cotton fabric exhibits an excellent antibacterial property and laundering durability. Its bacterial reduction rates (BR) against both $S$. aureus and E. coli remained over $97 \%$ even after 90 consecutive laundering cycles. Moreover, the modification causes insignificant damage to cotton's characteristics, such as tensile breaking strength, water absorptivity, and vapor permeability.

\section{Introduction}

Cotton fabric has suitable flexibility, water absorptivity, and air permeability for wearable textiles; therefore its wear comfortability has been well-received by customers..$^{1-3}$ To extend its market share, various efforts have been made to endow cotton textiles with additional functions, ${ }^{4-6}$ and such exploration has gradually concentrated on a few areas, for example, antibacterial effects, ${ }^{7,8}$ UV-protection, ${ }^{9,10}$ hydrophobic coating, ${ }^{11,12}$ flame retardance, ${ }^{13-15}$ and wear-resistance. ${ }^{16}$ Among these functions, the antibacterial effect has received more significant attention because the bacterial problem of cotton textiles often seriously troubles users. The temperature, moisture, and nutrients in the cotton textiles generally meet the requirements for the growth and reproduction of a multitude of bacteria and fungi ${ }^{17}$ and the microorganisms living on cotton fabrics often produce terrible odours, and cause colour degradation and even serious risks to human health. ${ }^{18,19}$ Therefore, a wide range of researchers have

${ }^{a}$ College of Materials and Textile, Zhejiang Sci-Tech University, Xiasha Higher Education Zone, Hangzhou 310018, China. E-mail: liuxd@zstu.edu.cn; Fax: +86571-86843785; Tel: +86-571-86843785

${ }^{b}$ State Key Laboratory for Diagnosis and Treatment of Infectious Diseases, Collaborative Innovation Center for Diagnosis and Treatment of Infectious Diseases, The First Affiliated Hospital, College of Medicine, Zhejiang University, 310003, Hangzhou, China.E-mail: diaohy@zju.edu.cn been putting efforts into antimicrobial cotton textiles, and numerous successful antibacterial cotton products have been applied in textile commodities including underwear, socks, curtains, and medical textile supplies. However, washing durability is still a practical challenge for most of the antimicrobial products, ${ }^{20-24}$ especially for the cotton fabrics modified using silver nanoparticles (Ag NPs). ${ }^{25}$

$\mathrm{Ag}$ NPs have strong antimicrobial effect, ${ }^{26-30}$ but they were suspected to have potential risks on the environment and human health. ${ }^{31,32}$ Moreover, the absence of covalent bonds between Ag NPs and cotton fibers makes the Ag NPs easy to split form the fiber surface during washing process. In this respect, various approaches have been investigated to enhance the adhesive force of Ag NPs onto cotton fabrics. As a result, it was reported that several ways, including polymer binders, ${ }^{33,34}$ composite nanoparticles, ${ }^{35,36}$ and surface modifications of cotton fiber $^{37}$ are effective on improving the antibacterial durability. Generally, these methods can give cotton fabrics with a certain degree of durability, for example, keeping more than $90 \%$ of the original antibacterial efficiency after $20-50$ washing cycles. ${ }^{38-40}$ However, achieving a satisfactory antibacterial durability with a minor dosage of Ag NPs still remains challenging. We have demonstrated that L-cysteine has an excellent effect on binding Ag NPs onto cotton fiber surface recently. ${ }^{41}$ Basing on the finding, we further pay attention to 
a similar amino acid, L-methionine, because it is one of the essential amino acids for human body and it has low toxicity towards mammalian cells as L-cysteine, but the electron donating effect of the methyl group may induce a stronger affinity with Ag NPs than L-cysteine does. The reducing agent used in this experiment is sodium borohydride, and its reduction product is sodium borate, which can be used as cleaning agents and cosmetics. It is unharmful to human being, and has little environmental risk.

In the present work, L-methionine is covalently linked onto cotton fiber surface via an esterification reaction with the hydroxyl groups of cellulose using a facile finishing pad-drycure process. Then Ag NPs are synthesized and immobilized on the modified surface by the enhanced adhesive force of the $\mathrm{L}^{-}$ methionine moieties. These reactions are monitored using a variety of analytical tools, including field emission scanning electron microscopy (FE-SEM), Fourier transform infrared spectroscopy (FTIR), X-ray diffraction (XRD), and X-ray photoelectron spectroscopy (XPS). Moreover, antibacterial effect and antibacterial durability of the finished fabrics are evaluated. As a result, L-methionine gives cotton fabrics with excellent laundering durability but without significant damages on cotton natures such as water absorptivity and vapor permeability. To our best knowledge, this is the first time to apply L-methionine as a binder to immobilize Ag NPs onto cotton fabrics, which offers outstanding antibacterial durability against washing.

\section{Materials and methods}

\subsection{Materials}

Cotton fabrics (abbreviated as Cot, warp density 60 stick per $\mathrm{cm}$, weft density 30 stick per $\mathrm{cm}$, thickness $0.42 \mathrm{~mm}$, square meter quality $120 \mathrm{~g} \mathrm{~m}^{-2}$, specific area $35.2 \mathrm{~m}^{2} \mathrm{~g}^{-1}$ ) were purchased from Shaoxing Qidong Textile Co., Ltd. (China). Before chemical modification, the cotton $(30 \mathrm{~mm} \times 30 \mathrm{~mm})$ fabrics were cleaned as previous literature. ${ }^{3} \mathrm{~L}$-Methionine and other reagents were purchased from shanghai Aladdin Co., Ltd (China) and used without further purification.

\subsection{Surface modification}

As shown in Scheme 1, a cotton fabric $(30 \mathrm{~mm} \times 30 \mathrm{~mm})$ was immersed in an aqueous solution of L-methionine $(100 \mathrm{~mL}$, $1.0 \mathrm{wt} \%$ ) for $30 \mathrm{~min}$, squeezed using a roller to a wet pick up of $90 \pm 2 \mathrm{wt} \%$, heated at $180^{\circ} \mathrm{C}$ for $5 \mathrm{~min}$, rinsed with deionized water $(100 \mathrm{~mL} \times 3$ times $)$ to remove unreacted $\mathrm{L}$-methionine molecules, and dried at $100{ }^{\circ} \mathrm{C}$ for $30 \mathrm{~min}$ to obtain the modified fabric (Me-Cot). The Me-Cot fabric was soaked in a solution

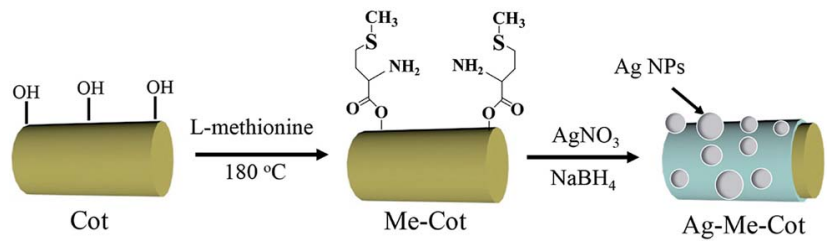

Scheme 1 Scheme of grafting L-methionine and forming Ag NPs on cotton fabric. of silver nitrate $\left(100 \mathrm{~mL}, 0.046 \mathrm{~mol} \mathrm{~L}^{-1}\right)$, heated at $100{ }^{\circ} \mathrm{C}$ for $30 \mathrm{~min}$, further soaked in a solution of sodium borohydride $\left(100 \mathrm{~mL}, 0.095 \mathrm{~mol} \mathrm{~L}^{-1}\right)$, rinsed with deionized water $(100 \mathrm{~mL} \times$ 3 times), and dried at $100{ }^{\circ} \mathrm{C}$ for $30 \mathrm{~min}$ to obtain the final cotton fabric sample (Ag-Me-Cot).

\subsection{Characterizations}

FTIR measurements were performed on a Nicolet Avatar 370 spectrometer (Nicolet Company, Madison, USA) with an attenuated total reflection (ATR) accessory. XRD analyses were obtained using an X-ray diffractometer (ARL XTRA, Switzerland) with $\mathrm{Cu} \mathrm{K}$ radiation $(\lambda=1.5418 \AA)$ operating at $40 \mathrm{kV}, 60 \mathrm{~mA}$, and the detailed protocol was described in our previous report. ${ }^{3}$ XPS analysis was performed by an AXIS multifunctional X-ray photoelectron spectrometer (ULTRA DLD, Shimadzu Ltd., Japan) at a power of $450 \mathrm{~W}$. Surface morphology of the modified cotton fabrics was investigated using a FE-SEM set (Ultra-55, Zeiss, Germany). Size distribution of the Ag NPs on fiber surface was calculated by measuring 200 bright points in the SEM images. The silver content in the finished cotton fabrics was measured by using inductively coupled plasma mass spectrometry (ICP-MS, Agilent 7500a, Australia).

Escherichia coli (E. coli, ATCC 1555) and Staphylococcus aureus (S. aureus, ATCC 547) were used as the model microorganisms, and the detailed protocol was described in our previous report. ${ }^{42}$

Laundering durability was evaluated by monitoring the antimicrobial ability of the cotton fabric sample periodically after every stringent washing process. ${ }^{42}$ For one laundering cycle, the cotton fabric $(15 \mathrm{~mm} \times 15 \mathrm{~mm})$ was washed with $50 \mathrm{~mL}$ of an aqueous solution of sodium dodecanesulphonate $(2.0 \%, \mathrm{w} / \mathrm{w})$ in a beaker (diameter, $50 \mathrm{~mm}$ ) with stirring (300 rpm, magnetic stirrer, $9 \mathrm{~mm} \times 25 \mathrm{~mm}$ ) at $25^{\circ} \mathrm{C}$.

As our previous work, ${ }^{42}$ water absorptivity was measured by weighting the difference of the cotton samples after soaking in deionized water and hanging out, water vapor permeability was evaluated by using the ASTM E-96 (open cup test) method, ${ }^{43}$ tensile strength tests were carried out on an electronic fabric tensile tester (YG065, China) at a constant rate of $20 \mathrm{~cm} \mathrm{~min}^{-1}$.

\section{Results and discussion}

\subsection{Preparation and characterization of the Ag-Me-Cot cotton fabric}

Pad-dry-cure process was used to graft L-methionine on the surface of cotton fibers and in situ-synthesize Ag NPs on the modified surface (Scheme 1). Pad-dry-cure method is a very usual technique can be found in almost all dyeing and finishing factories. To perform reactions on fabrics, it generally contains four successive steps: dipping fabric in a functional solution, squeezing to control the wet weight, drying and curing to promote the reaction rate. The reaction conditions (such as heating at $180^{\circ} \mathrm{C}$ for more than $2 \mathrm{~min}$ ) for esterification with the hydroxyl groups of cotton fabric have been well established. ${ }^{\mathbf{4 4 , 4 5}}$

Fig. 1 compares the ATR-FTIR spectra of Cot (a), Me-Cot (b) and Ag-Me-Cot (c). Both Me-Cot and Ag-Me-Cot exhibit new peaks at $1725 \mathrm{~cm}^{-1}$ after the modification process. The peak is 


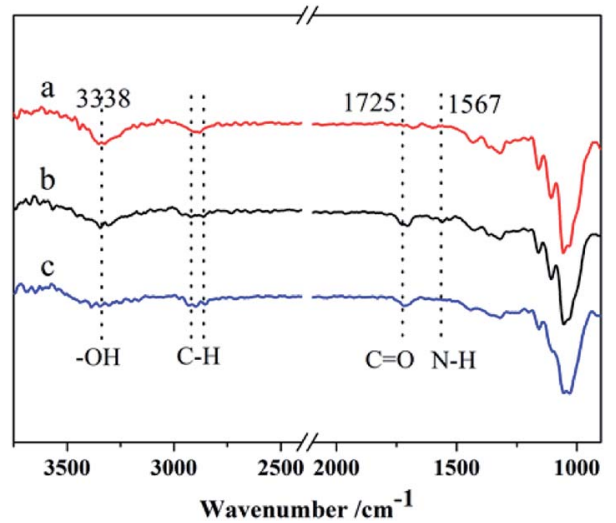

Fig. 1 ATR-FTIR spectra of the Cot (a), Me-Cot fabric (b), and Ag-MeCot fabric (c).

associable to the asymmetric stretching of $\mathrm{COOH}$ groups and COOR groups, implying that the esterification reactions occurred between the L-methionine and the hydroxyl groups of cellulose on cotton surface. ${ }^{46}$ Additionally, by comparing the spectra of Cot and Me-Cot, the attenuation observed on the peak around $3340 \mathrm{~cm}^{-1}$ (for $-\mathrm{OH}$ ) and appearance of new peaks around $1567 \mathrm{~cm}^{-1}$ (for $-\mathrm{NH}_{2}$ ) further suggest the introduction

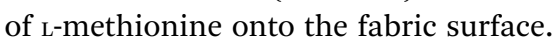

Fig. 2 shows the XRD spectra of Cot (a) and Ag-Me-Cot (b). The peaks appearing at $2 \theta=39,45,65$, and 78 are assigned to the Ag (111), (200), (220), and (311) planes, respectively. As reported before, ${ }^{7,8,46,47}$ they are typical XRD peaks of Ag NPs having a pure crystalline structure. Moreover, by applying the DebyeScherrer equation, ${ }^{48}$ the average size of the $\mathrm{Ag}$ NPs loaded on the fabric is calculated as $83.4 \mathrm{~nm}$.

Fig. $3 \mathrm{a}$ and $\mathrm{b}$ show the wide-range XPS spectra of Cot and AgMe-Cot fabric samples. Cot displays C 1s and O 1s signals, but AgMe-Cot has additional S $2 \mathrm{p}$ and $\mathrm{Ag}$ 3d signals. The high resolution XPS C 1s spectra of Cot and Ag-Me-Cot are deconvoluted in Fig. 3c and d, respectively. The $\mathrm{C}$ 1s peak for Cot can be decomposed to two peaks with binding energy of $284 \mathrm{eV}(\mathrm{C}-\mathrm{C})$ and $286 \mathrm{eV}(\mathrm{C}-\mathrm{OH})$. Whereas in the case of Ag-Me-Cot fabric, the $\mathrm{C}$ 1s peak compose of new peaks at $287.2 \mathrm{eV}$ and $288.8 \mathrm{eV}$, which are assignable to $\mathrm{C}-\mathrm{S}$ and $\mathrm{C}=\mathrm{O}$ bonds, ${ }^{49}$ respectively. Fig. 3e shows the $\mathrm{Ag} 3 \mathrm{~d}$ XPS spectra of Ag-Me-Cot, and the peaks at $368.0 \mathrm{eV}$ and $374.0 \mathrm{eV}$ can

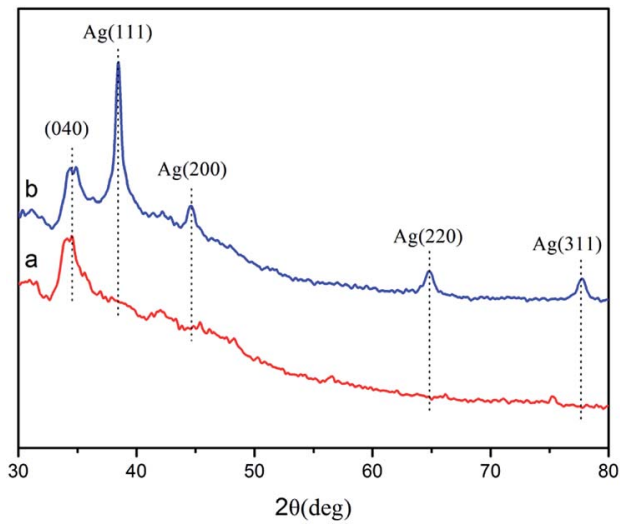

Fig. 2 XRD patterns of the Cot (a), and Ag-Me-Cot fabric (b).
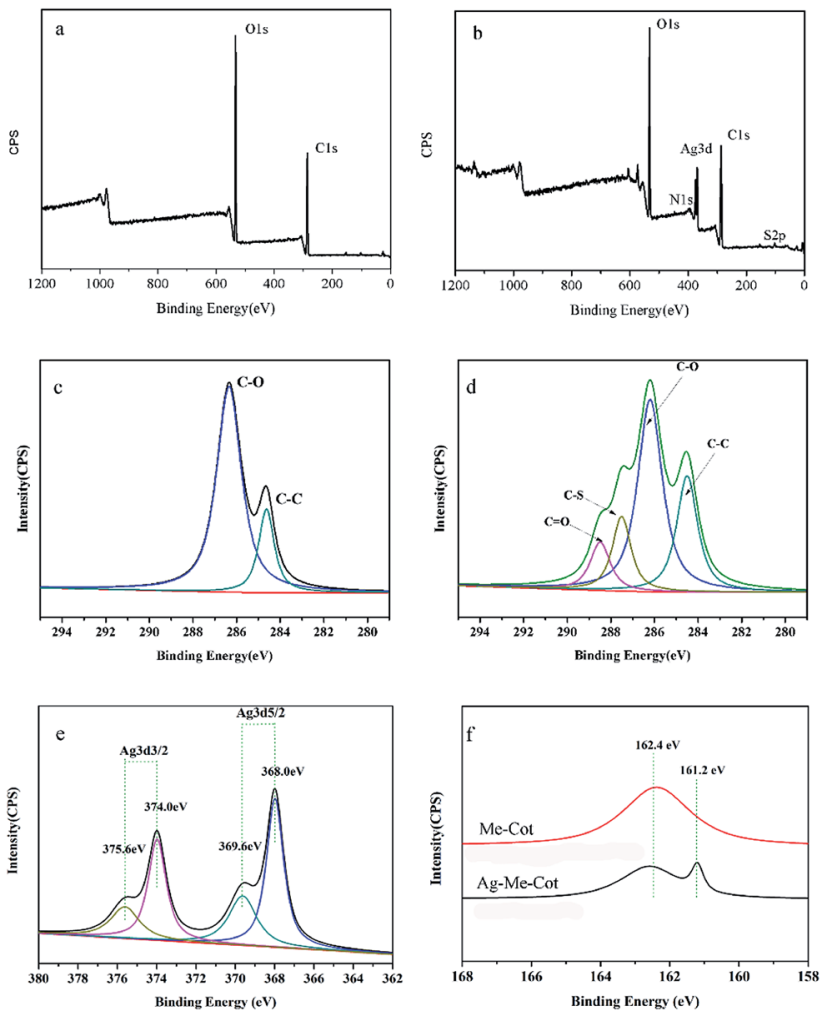

Fig. 3 XPS spectra of the cotton fabrics. Wide-range and high resolution C 1s XPS spectra of Cot (a and c) and Ag-Me-Cot fabric (b and d); high resolution $\mathrm{Ag} 3 \mathrm{~d}$ XPS spectrum of $\mathrm{Ag}-\mathrm{Me}-\mathrm{Cot}$ fabric (e) and high resolution S 2p XPS spectra of Me-Cot and Ag-Me-Cot fabrics (f).

be attributed to $\mathrm{Ag}^{0}$. The $6.0 \mathrm{eV}$ slitting between the two peaks, $\mathrm{Ag}$ (3d5/2) and $\mathrm{Ag}(3 \mathrm{~d} 3 / 2)$, indicates the existence form of metallic silver on the fabric surface.$^{50}$ Moreover, it is very obvious that these peaks can be further decomposed to two peaks with a $1.6 \mathrm{eV}$ slitting of binding energy. Because $\mathrm{Ag}^{+}$shows lower binding energy than $\mathrm{Ag}^{0}{ }^{0},{ }^{51,52}$ the attribution of the split peaks to silver ion can be disconfirmed. The positive shifts of $1.6 \mathrm{eV}$ suggest the existence of small Ag NPs on the cotton fiber surface. Typically, 0.5-2.0 eV shifts have been observed previously for small nanoparticles compared to peaks of bulk crystals. ${ }^{53}$ The coordination bonds of Ag atoms with $\mathrm{L}$-methionine binder is verified by the $\mathrm{S} 2 \mathrm{p}$ spectra shown in Fig. 3f. By comparing with the modified cotton before loading Ag NPs, the new peak appeared at $161.2 \mathrm{eV}$ is attributable to the highly de-electronated state of $\mathrm{S}$ elements. ${ }^{54}$ These XPS results suggest that the L-methionine molecules have been covalently linked on the fiber surface by ester groups, and there are coordination bonds between the Ag NPs and the S atoms of Lmethionine.

Fig. 4 shows the SEM images of the cotton fabric surfaces. Cot surface is clean and smooth (Fig. 4a), but the modified cotton surfaces are rough (Fig. $4 \mathrm{~b}-\mathrm{d}$ ). The fiber surface of MeCot seems covered by a layer of material (Fig. $4 \mathrm{~b}$ ), and the fiber surfaces of Ag-Me-Cot before and after washing (Fig. 4c and d) have numerous particles (Ag NPs).

These SEM results indicate that a lot of Ag NPs were remained on the Ag-Me-Cot surfaces even after 90 laundering cycles. The diagrams of the size distribution (calculated via the 


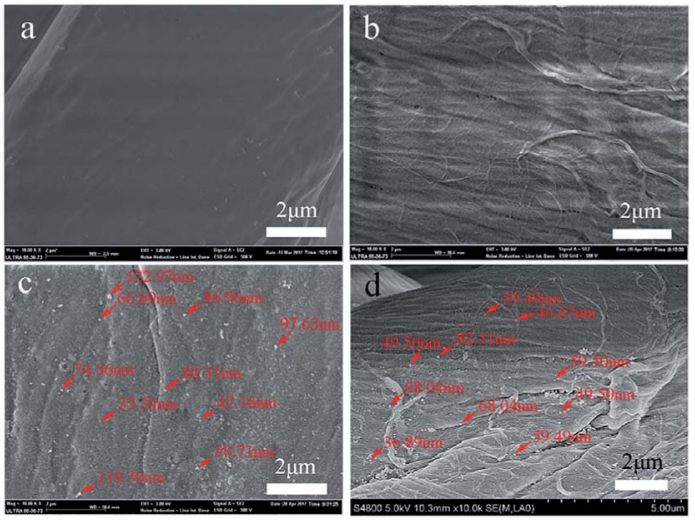

Fig. 4 SEM images of the fiber surfaces. (a) Cot fiber, (b) Me-Cot, (c) $\mathrm{Ag}-\mathrm{Me}-\mathrm{Cot}$, and (d) Ag-Me-Cot after 90 washing cycles.

statistical analyses) of the Ag NPs are given in Fig. 5a and b, indicating that the Ag NPs loaded on the fiber surface of Ag-MeCot became smaller by the washing action. Moreover, the size range of the Ag NPs became more narrow (ranged from 10 to $110 \mathrm{~nm}$, with an average diameter of $47.75 \mathrm{~nm}$, following a normal distribution) after washed for 90 laundering cycles when compared with the Ag-Me-Cot fabric before washing (ranged from 10 to $170 \mathrm{~nm}$, with an average diameter of $84.13 \mathrm{~nm}$, following a positive skewed distribution). This result means that larger Ag NPs are easier to loss from the modified cotton fabrics.

To further examine the quantities of Ag NPs on the cotton surface, ICP-MS technique was carried out on the cotton fabrics. As shown in Table 1, after 90 laundering tests, the silver content of the Ag-Me-Cot fabric was reduced by $10.2 \%$, which in good
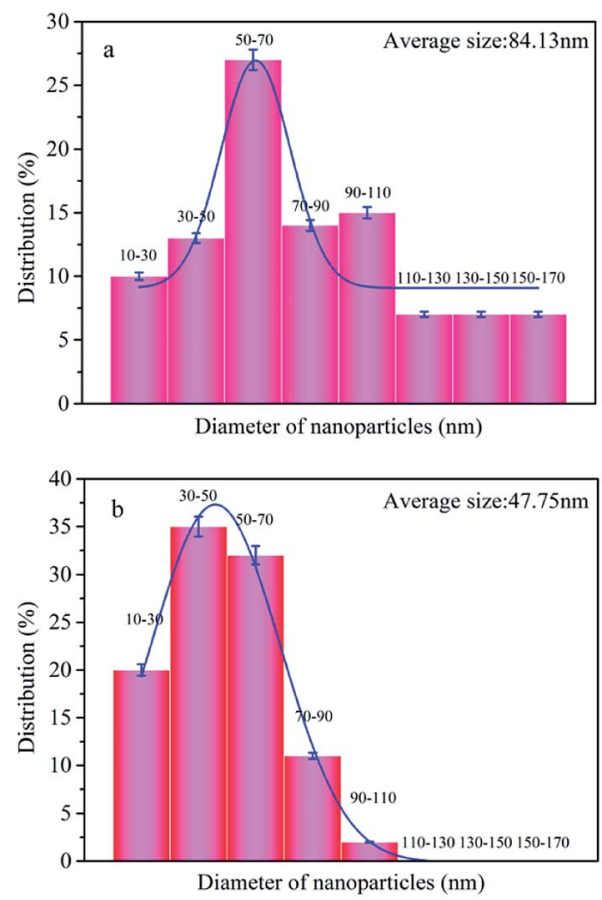

Fig. 5 Size distribution of the Ag NPs loaded on the cotton fabrics. (a) $\mathrm{Ag}-\mathrm{Me}-\mathrm{Cot}$ and (b) Ag-Me-Cot after 90 washing cycles.
Table 1 ICP-MS analysis results of the silver content and the antibacterial effect of the cotton fabrics

\begin{tabular}{lllll}
\hline & & \multicolumn{2}{l}{$\begin{array}{l}\text { Bacterial reduction } \\
\text { rate (\%) }\end{array}$} \\
\cline { 3 - 5 } Sample & $\mathrm{Ag}\left(\mathrm{mg} \mathrm{kg}^{-1}\right)$ & $\begin{array}{l}\text { Remaining } \\
\text { rates of } \mathrm{Ag}(\%)\end{array}$ & E. coli & S. aureus \\
\hline $\begin{array}{l}\text { Ag-Me-Cot } \\
\begin{array}{l}\text { Ag-Me-Cot } \\
\text { washed }\end{array}\end{array}$ & 250 & - & $99.0 \pm 1.0$ & $99.0 \pm 1.0$ \\
${ }^{a}$ After washed for 90 laundering tests. & $99.0 \pm 1.0$ & $98.0 \pm 1.0$ \\
\end{tabular}

agreement with the size reduction of the Ag NPs. These results suggest that larger Ag particles are more easily washed off from the fiber surface.

\subsection{Antibacterial efficacy and laundering durability}

Fig. 6 shows the optical images of inhibition zones towards $E$. coli and S. aureus. Cot and Me-Cot fabric have no obvious bacteriostatic effect as the diameters of their inhibition zones are constant at $1.00 \mathrm{~cm}$. However, in case of Ag-Me-Cot fabric, the antibacterial diameters towards E. coli and $S$. aureus reached to $1.74 \mathrm{~cm}$ (Fig. 6Ec) and $1.70 \mathrm{~cm}$ (Fig. 6Sc), respectively. This indicates that the bacteriostatic effect is caused by the loading of the Ag NPs.

Fig. 7 shows the antimicrobial durability of the modified cotton fabrics. Although it was washed by a certain number of laundering cycles, its BR rates for both bacteria are kept at $100 \%$. Even after 90 washing cycles, as shown in Fig. 7, the BR rates are remained over $97.7 \%$. In other reports, ${ }^{7,55-57}$ the general durability level is that a modified cotton fabric maintains $90 \%$ BR value after 10-50 laundering cycles. Therefore, the antimicrobial results demonstrated here have significant improvement on washing durability by comparing with previous reports.

As shown in the Table 1, the loss quantity of the Ag NPs $\left(0.11 \%, 0.33 \mathrm{mg} \mathrm{kg}^{-1}\right.$ per once laundering) is better than that reported in previous works. In addition, the silver content is about $250 \mathrm{mg} \mathrm{kg}^{-1}$ cotton fabric, which is far smaller than that reported early before. ${ }^{58-60}$ The improvement on immobilization of $\mathrm{Ag}$ NPs can be contributed to the linking effect of $\mathrm{L}^{-}$ methionine.

In our earlier study, it was reported that $97.6 \% \mathrm{BR}$ value and $10 \%$ silver loss after 50 laundering cycles can be achieved by the linking effect of L-cysteine. ${ }^{41}$ In the present work, almost same BR level (higher than 98\%) and 10\% silver loss are kept even

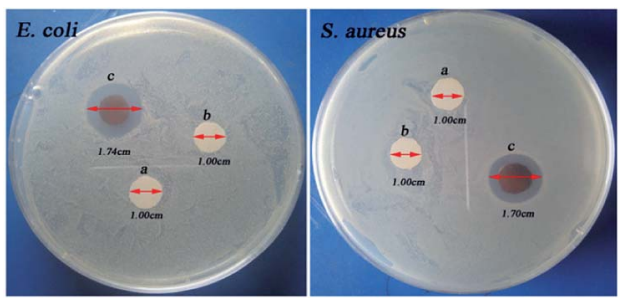

Fig. 6 The optical images of the inhibition zone tests. (a) Cot, (b) MeCot, and (c) Ag-Me-Cot. 
(a)
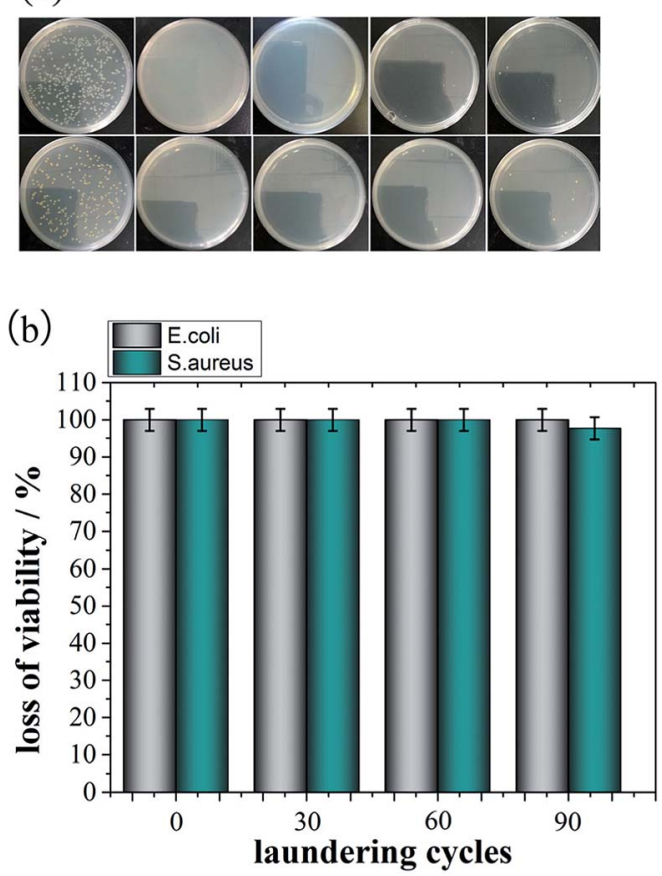

Fig. 7 Antibacterial durability of Ag-Me-Cot fabric. (a) The optical images of the ager plates after the colony-forming tests, and (b) the BR rates against washing cycles.

Table 2 Important natures of the cotton fabrics

\begin{tabular}{llll}
\hline $\begin{array}{l}\text { Cotton } \\
\text { sample }\end{array}$ & $\begin{array}{l}\text { Ventilation } \\
\text { property }\left(\mathrm{g} \mathrm{m}^{-2} \mathrm{~d}^{-1}\right)\end{array}$ & $\begin{array}{l}\text { Water } \\
\text { absorption (\%) }\end{array}$ & $\begin{array}{l}\text { Tensile } \\
\text { strength (MPa) }\end{array}$ \\
\hline Cot & $1560 \pm 9.0$ & $246.0 \pm 22.0$ & $17.5 \pm 0.2$ \\
Ag-Me-Cot & $1520 \pm 13.0$ & $238.0 \pm 14.0$ & $22.6 \pm 0.3$
\end{tabular}

until 90 consecutive laundering cycles were finished. Thus, we conclude that the outstanding antibacterial durability with fewer silver loading achieved in the present work can be contributed to the stronger affinity of L-methionine, which has an additional electron donating effect of the methyl group.

\subsection{Influences on the intrinsic cotton natures}

Significant damages on cotton natures are generally unacceptable. As shown in the Table 2, Ag-Me-Cot shows good vapor permeability, water absorbability, and tensile breaking strength, which very closed to that of Cot. These results indicate that the desired cotton natures are largely preserved after grating $\mathrm{L}$-methionine binder and forming $\mathrm{Ag}$ NPs on cotton fabrics.

\section{Conclusions}

L-Methionine can improve adhesive force of Ag NPs onto fiber surface of cotton. The binding effect of L-methionine gives cotton fabrics with a remarkable antibacterial durability that can keep the BR rate more than $97 \%$ even after 90 stringent laundering cycles. It is notable that such good antibacterial durability is obtained by a small amount of silver loading. The high washing durability is contributable to the coordination bonds between Ag NPs and the L-methionine moieties that grafted on the cotton fiber surface. Importantly, our finishing method does not significantly damage cotton natures such as tensile breaking strength, water absorptivity, and vapor permeability. It is believed that the finishing method has great potential for practical applications in biomedical textiles.

\section{Conflicts of interest}

The authors have no conflicts to declare.

\section{Acknowledgements}

This work was financially supported by the Natural Science Foundation of China (51573167) and Public Welfare Technology Application Research Project of Zhejiang Province (2017C31035 and 2017C33154).

\section{Notes and references}

1 D. Saravanan, N. S. Vasanthi and T. Ramachandran, Carbohydr. Polym., 2009, 76, 1-7.

2 Q. Xu, L. Xie, H. Diao, F. Li, Y. Zhang, F. Fu and X. Liu, Carbohydr. Polym., 2017, 177, 187-193.

3 A. C. Baker, Agric. Hist., 2015, 89, 119-121.

4 B. Cortese, D. Caschera, G. Padeletti, G. M. Ingo and G. Gigli, Surf. Innovations, 2013, 1, 140-156.

5 A. K. Yetisen, Q. Hang, A. Manbachi, H. Butt, M. R. Dokmeci, J. P. Hinestroza, M. Skorobogatiy, A. Khademhosseini and S. H. Yun, ACS Nano, 2016, 10, 3042-3068.

6 H. E. Emam, N. H. Saleh, K. S. Nagy and M. K. Zahran, Int. J. Biol. Macromol., 2016, 84, 308-318.

7 Y. Gao and R. Cranston, Text. Res. J., 2010, 60, 60-72.

8 M. M. G. Fouda, E. S. Abdel-Halim and S. S. Al-Deyab, Carbohydr. Polym., 2013, 92, 943-954.

9 S. Muzaffar, I. A. Bhatti, M. Zuber, H. N. Bhatti and M. Shahid, Int. J. Biol. Macromol., 2017, 94, 51-60.

10 O. K. Alebeid and T. Zhao, J. Text. Inst., 2017, 108, 2027-2039.

11 G. H. Xi, W. C. Fan, L. Wang, X. D. Liu and T. Endo, J. Polym. Sci., Part A: Polym. Chem., 2015, 53, 1862-1871.

12 L. Wang, G. H. Xi, S. J. Wan, C. H. Zhao and X. D. Liu, Cellulose, 2014, 21, 2983-2994.

13 T. Liang, Z. Jiang, C. Wang and J. Liu, J. Appl. Polym. Sci., 2017, 134, 45114-45119.

14 H. Yan, L. Zhao, Z. Fang and H. Wang, J. Appl. Polym. Sci., 2017, 134, 45556.

15 Z. W. Yang, Y. Y. Zhang, F. Y. Fu and X. D. Liu, RSC Adv., 2017, 7, 53871-53877.

16 W. Fan, Y. Zhu, G. Xi, M. Huang and X. D. Liu, J. Appl. Polym. Sci., 2016, 133, 43024-43030.

17 Y. Zhang, Q. Xu, F. Fu and X. D. Liu, Cellulose, 2016, 23, 2791-2808.

18 X. Dai, Z. Fan, Y. Lu and P. C. Ray, ACS Appl. Mater. Interfaces, 2013, 5, 11348-11354. 
19 C. M. Courtney, S. M. Goodman, J. A. McDaniel, N. E. Madinger, A. Chatterjee and P. Nagpal, Nat. Mater., 2016, 15, 529-534.

20 M. Salat, P. Petkova, J. Hoyo, I. Perelshtein, A. Gedanken and T. Tzanov, Carbohydr. Polym., 2018, 189, 198-203.

21 J. Lin, X. Chen, C. Chen, J. Hu, C. Zhou, X. Cai, W. Wang, C. Zheng, P. Zhang and J. Cheng, ACS Appl. Mater. Interfaces, 2018, 10, 6124-6136.

22 L. Li, X. Chi, F. Gai, H. Zhou, F. Zhang and Z. Zhao, J. Appl. Polym. Sci., 2017, 134, 45323.

23 G. Luo, G. Xi, X. Wang, D. Qin, Y. Zhang, F. Fu and X. Liu, J. Appl. Polym. Sci., 2017, 134, 44897-44903.

24 G. Xi, Y. Xiu, L. Wang and X. Liu, J. Appl. Polym. Sci., 2015, 132, 41824-41830.

25 G. Sun, J. Chem. Educ., 2005, 82, 5.

26 D. A. Mosselhy, H. Granbohm, U. Hynonen, Y. Ge, A. Palva, K. Nordstrom and S. P. Hannula, Nanomaterials, 2017, 7, 7090261-7090279.

27 T. T. T. Vi, S. Rajesh Kumar, B. Rout, C. H. Liu, C. B. Wong, C. W. Chang, C. H. Chen, D. W. Chen and S. J. Lue, Nanomaterials, 2018, 8, 8030162-8030177.

28 S. Gomez-Grana, M. Perez-Ameneiro, X. Vecino, I. PastorizaSantos, J. Perez-Juste, J. M. Cruz and A. B. Moldes, Nanomaterials, 2017, 7, 7060139-7060153.

29 F. Tanvir, A. Yaqub, S. Tanvir and W. A. Anderson, Nanomaterials, 2017, 7, 7100296-7100310.

30 M. C. Sportelli, R. A. Picca, F. Paladini, A. Mangone, L. C. Giannossa, C. D. Franco, A. L. Gallo, A. Valentini, A. Sannino, M. Pollini and N. Cioffi, Nanomaterials, 2017, 7, 7080203-7080218.

31 S. León-Silva, F. Fernández-Luqueño and F. López-Valdez, Water, Air, Soil Pollut., 2016, 227, 305-325.

32 Y. Li, Y. Zhang and B. Yan, Int. J. Mol. Sci., 2014, 15, 36713697.

33 H. Xu, X. Shi, H. Ma, Y. Lv, L. Zhang and Z. Mao, Appl. Surf. Sci., 2011, 257, 6799-6803.

34 D. Zhang, L. Chen, C. Zang, Y. Chen and H. Lin, Carbohydr. Polym., 2013, 92, 2088-2094.

35 W. Zhu, M. Kuang, S. Wei, T. Duan, Y. Wang, D. Zhou, L. Ma, D. Fang, J. Zhou and W. Yang, J. Exp. Nanosci., 2015, 11, 471479.

36 Y. Di, Q. Li and X. Zhuang, J. Eng. Fibers Fabr., 2012, 7, 24-29.

37 M. K. Zahran, H. B. Ahmed and M. H. El-Rafie, Carbohydr. Polym., 2014, 108, 145-152.

38 M. Montazer, F. Alimohammadi, A. Shamei and M. K. Rahimi, Colloids Surf., B, 2012, 89, 196-202.
39 M. Gouda, A. Aljaafari, Y. Al-Fayz and W. E. Boraie, J. Nanomater., 2015, 16, 1-9.

40 L. M. Liu and B. Deng, Sci. Rep., 2014, 4, 5920-5929.

41 Q. Xu, J. Gu, Y. Zhao, X. Ke and X. D. Liu, Fibers Polym., 2017, 18, 2204-2211.

42 Q. B. Xu, Y. H. Wu, Y. Y. Zhang, F. Y. Fu and X. D. Liu, Fibers Polym., 2016, 17, 1782-1789.

43 Q. Xu, X. Ke, L. Shen, N. Ge, Y. Zhang, F. Fu and X. D. Liu, Int. J. Biol. Macromol., 2018, 111, 796-803.

44 H. Qi, Y. Huang, B. Ji, G. Sun, F. L. Qing, C. Y. Hu and K. L. Yan, Carbohydr. Polym., 2016, 135, 86-93.

45 C. Schramm, B. Rinderer and R. Tessadri, Carbohydr. Polym., 2014, 105, 81-89.

46 Q. Xu, X. Ke, D. Cai, Y. Zhang, F. Fu, T. Endo and X. D. Liu, Cellulose, 2018, 25, 2129-2141.

47 V. Prysiazhnyi, A. Kramar, B. Dojcinovic, A. Zekic, B. M. Obradovic, M. M. Kuraica and M. Kostic, Cellulose, 2013, 20, 315-325.

48 M. Zhang, J. Pang, W. Bao, W. Zhang, H. Gao, C. Wang, J. Shi and J. Li, Appl. Surf. Sci., 2017, 419, 16-23.

49 J. Wang, G. Xi, G. Luo and X. D. Liu, Cellulose, 2016, 23, 915927.

50 W.-G. Kwak, M. H. Oh and M.-S. Gong, Carbohydr. Polym., 2015, 115, 317-324.

51 X.-Y. Gao, S.-Y. Wang, J. Li, Y.-X. Zheng, R.-J. Zhang, P. Zhou, Y.-M. Yang and L.-Y. Chen, Thin Solid Films, 2004, 455-456, 438-442.

52 D. Wang, Y. Duan, Q. Luo, X. Li and L. Bao, Desalination, 2011, 270, 174-180.

53 I. Lopez-Salido, D. C. Lim and Y. D. Kim, Surf. Sci., 2005, 588, 6-18.

54 F. Porcaro, L. Carlini, A. Ugolini, D. Visaggio, P. Visca, I. Fratoddi, I. Venditti, C. Meneghini, L. Simonelli, C. Marini, W. Olszewski, N. Ramanan, I. Luisetto and C. Battocchio, Materials, 2016, 9, 1-15.

55 A. Hebeish, A. El-Shafei, S. Sharaf and S. Zaghloul, Carbohydr. Polym., 2011, 84, 605-613.

56 N. A. Ibrahim, B. M. Eid, T. M. Elmaaty and E. A. Elaziz, Carbohydr. Polym., 2013, 94, 612-618.

57 X. Yue, H. Lin, T. Yan, D. Zhang, H. Lin and Y. Chen, Fibers Polym., 2014, 15, 716-722.

58 T. Jiang, L. Liu and J. Yao, Fibers Polym., 2011, 12, 620-625. 59 H. Liu, Y.-Y. Lee, T. B. Norsten and K. Chong, J. Ind. Text., 2013, 44, 198-210.

60 D. Klemenčič, B. Tomšič, F. Kovač, M. Žerjav, A. Simončič and B. Simončič, J. Mater. Sci., 2014, 49, 3785-3794. 